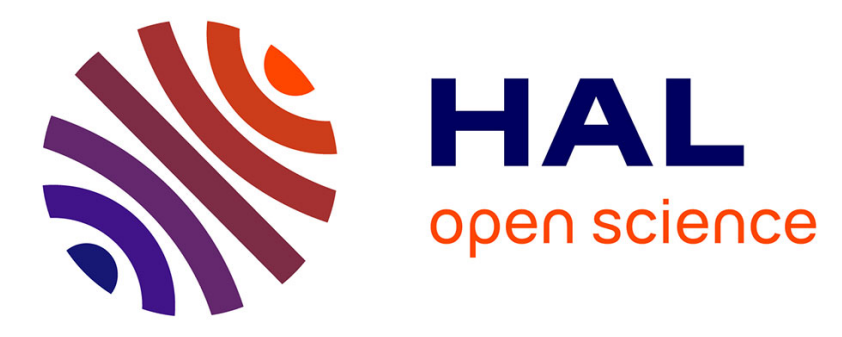

\title{
On the choice of parameters in the phase field method for simulating crack initiation with experimental validation
}

T.T. Nguyen, Julien Yvonnet, Michel Bornert, Camille Chateau, Karam Sab, R. Romani, Robert Le Roy

\section{To cite this version:}

T.T. Nguyen, Julien Yvonnet, Michel Bornert, Camille Chateau, Karam Sab, et al.. On the choice of parameters in the phase field method for simulating crack initiation with experimental validation. International Journal of Fracture, 2016, 197 (2), pp.213-226. 10.1007/s10704-016-0082-1 . hal01258035

\section{HAL Id: hal-01258035 \\ https://hal.science/hal-01258035}

Submitted on 18 Jan 2016

HAL is a multi-disciplinary open access archive for the deposit and dissemination of scientific research documents, whether they are published or not. The documents may come from teaching and research institutions in France or abroad, or from public or private research centers.
L'archive ouverte pluridisciplinaire $\mathbf{H A L}$, est destinée au dépôt et à la diffusion de documents scientifiques de niveau recherche, publiés ou non, émanant des établissements d'enseignement et de recherche français ou étrangers, des laboratoires publics ou privés. 


\title{
On the choice of parameters in the phase field method for simulating crack initiation with experimental validation
}

\author{
T.T. Nguyen - J. Yvonnet - M. \\ Bornert - C. Chateau - K. Sab - R. \\ Romani · R. Le Roy
}

Received: date / Accepted: date

\begin{abstract}
The phase field method is a versatile simulation framework for studying initiation and propagation of complex crack networks without dependence to the finite element mesh. In this paper, we discuss the influence of parameters in the method and provide experimental validations of crack initiation and propagation in plaster specimens. More specifically, we show by theoretical and experimental analyses that the regularization length should be interpreted as a material parameter, and identified experimentally as it. Qualitative and quantitative comparisons between numerical predictions and experimental data are provided. We show that the phase field method can predict accurately crack initiation and propagation in plaster specimens in compression with respect to experiments, when the material parameters, including the characteristic length are identified by other simple experimental tests.
\end{abstract}

Keywords Crack initiation · Crack propagation · Simulation · Plaster · Digital image correlation

\section{Introduction}

Simulation of crack initiation and growth in brittle materials such as concrete, cement or rocks is a major concern for predicting the strength and durability of structures made of these materials. One issue lies in the prediction of the onset of cracks in brittle materials: the classical Griffith theory of brittle fracture fails

J. Yvonnet

Université Paris-Est, Laboratoire Modélisation et Simulation Multi Échelle MSME UMR 8208 CNRS, 5 bd Descartes, F-77454 Marne-la-Vallée, France.

E-mail: julien.yvonnet@univ-paris-est.fr

M. Bornert

Université Paris-Est, Laboratoire Navier, CNRS UMR8205, ENPC, IFSTTAR, 6 et 8 avenue Blaise Pascal, 77455 Marne-la-Vallée Cedex, France. 
to predict crack initiation in un-notched specimens [14]. On the other hand, damage models with softening suffer from strong drawbacks when implemented in numerical solving methods such as the finite element method, like mesh dependency and lack of convergence of the fracture energy as the element size goes to zero $[32,2]$. This has been shown to yield from a loss of ellipticity of the associated mechanical problem $[38,20,8]$. To circumvent these issues, regularization schemes must be applied, such as nonlocal damage models [33, 3] and higher-order deformation gradient schemes [30,23]. Another possibility is to introduce cohesive layers in the models which are then numerically solved via cohesive finite elements $[40,10,41]$. Cohesive elements require cracks to follow the element boundaries of the mesh. Other techniques like XFEM [27, 4,12 ] require a pre-existing crack and are not well adapted to complex cracks morphologies due to the underlying level-set functions needed to describe the displacement jump. Finally, we mention a new method, called Thick Level-Set method (TLS) $[6,11]$ in which a level-set function is employed to separate the undamaged zone from the damaged one, and where the crack is a consequence of the damage front motion, allowing crack initiation.

Recently, the phase field method has been proposed in $[9,13-17,25,37]$ (only to name a few). It employs a diffuse approximation of discontinuities related to cracks and is consistent with brittle fracture through a modified variational principle. This technique is able to simulate brittle crack initiation and propagation without dependence to the mesh in a classical FEM framework. It allows handling very complex, multiple crack fronts and branching in both $2 \mathrm{D}$ and $3 \mathrm{D}$ without ad hoc numerical treatment. In $[28,29]$, the authors have demonstrated the capability of the method to simulate crack onset and propagation in complex image-based models, as such obtained by segmenting 3D X-Ray computed tomography images of real materials like concrete.

However, the method requires choosing a regularization parameter related to the smeared approximation of discontinuities. This parameter induces a characteristic length $l$ in the model which must be chosen by the user. In [1], Amor et al. have shown that a relationship can be established between $l$ and at least two other material parameters. This seems consistent with a recent crack initiation criterion of Leguillon et al. [21] where two material parameters need to be identified for predicting crack onset. In the present work, we follow this line and show that $l$ may be interpreted as a material parameter and should be deduced from experimental material parameters identification when available. We validate this by comparing simulations of crack initiation with experiments on drilled plaster samples, where the material parameters, including $l$, have been identified in other simple experimental tests [35]. Experimental data provided in [35] have been used to provide reference solutions associated with onset of cracks in plaster structures containing drilled holes in compression or in three-point bending of a beam. We also discuss the influence of other numerical parameters on the solution provided by the phase field method such as the size of load increments and the mesh size. Note that complementary results related to this work can be found in a recent paper by Mary et al. [24]. 
In the following, we first give a brief summary of the phase field method in section 2 . In section 3 , we discuss the influence of the main parameters in the numerical method on the predicted mechanical response of cracked structures and show more specifically the relationship between the regularization parameter in the phase field method and some material parameters. In sections 4,5 and 6 , we provide qualitative and quantitative comparisons between experimental data of crack initiation in plaster samples with simulations.

\section{Mechanical model and numerical simulation method}

In the following, the basic concepts of the phase field method are briefly summarized. For more details and practical implementation aspects, the interested reader can refer to $[25,28]$. The phase field method is based on a regularized formulation of a sharp crack description. A regularized variational principle describing both the evolution of the mechanical problem and of an additional field $d$ describing the damage (called phase field), is discretized by a finite element procedure and a staggered algorithm, chosen here due to its easier implementation. The method alleviates the shortcomings of remeshing crack geometry by using a fixed mesh and a regularized description of the discontinuities. In addition, crack initiation can be modeled in a straightforward manner. In contrast to volume damage models, usually implemented in nonlinear codes, such regularized approach is directly connected to the brittle crack theory of crack propagation. In the present work, the phase field method has been implemented in a in-house code both in $2 \mathrm{D}$ and $3 \mathrm{D}$.

In the phase field method, assuming small strains, the regularized form of the energy describing the cracked structure is expressed by:

$$
E(\mathbf{u}, d)=\int_{\Omega} W(\mathbf{u}, d) d \Omega+g_{c} \int_{\Omega} \gamma(d) d \Omega,
$$

where $W$ is the density of the elastic energy, depending on the displacements $\mathbf{u}(\mathbf{x})$ and on the phase field $d(\mathbf{x})$ describing the damage of the solid, $g_{c}$ is the fracture resistance and $\gamma(d)$ is the crack energy density, defined by $\gamma(d, \nabla d)=$ $\frac{1}{2 l} d^{2}+\frac{l}{2} \nabla d \cdot \nabla d$ (see e.g. $\left.[25,28]\right)$.

Applying the principle of maximum dissipation and energy minimization [14] to (1) yields the set of coupled equations to be solved on the domain $\Omega$ associated with the structure, with boundary $\delta \Omega$ and outward normal $\mathbf{n}$, to determine $d(\mathbf{x})$ and $\mathbf{u}(\mathbf{x}), \forall \mathbf{x} \in \Omega$ :

$$
\left\{\begin{array}{l}
2(1-d) \mathcal{H}-\frac{g_{c}}{l}\left\{d-l^{2} \Delta d\right\}=0 \text { in } \Omega, \\
d(\mathbf{x})=1 \text { on } \Gamma, \\
\nabla d(\mathbf{x}) \cdot \mathbf{n}=0 \text { on } \partial \Omega
\end{array}\right.
$$

and

$$
\left\{\begin{array}{l}
\nabla \cdot \boldsymbol{\sigma}(\mathbf{u}, d)=\mathbf{f} \text { in } \Omega \\
\mathbf{u}(\mathbf{x})=\overline{\mathbf{u}} \text { on } \partial \Omega_{u} \\
\boldsymbol{\sigma} \mathbf{n}=\overline{\mathbf{F}} \text { on } \partial \Omega_{F}
\end{array}\right.
$$


In (2), $\Gamma$ refers to the crack surface, $l$ is the regularization parameter. The history strain energy density function $\mathcal{H}(t)$ is introduced to describe a dependence on history [25] and possible loading-unloading. This function reads:

$$
\mathcal{H}(\mathbf{x}, t)=\max _{\tau \in[0, t]}\left\{\Psi^{+}(\mathbf{x}, \tau)\right\},
$$

In (4), $\Psi^{+}$is the tensile part of the elastic strain density function serving to model unilateral contact. It is defined as

$$
\Psi^{+}(\varepsilon)=\frac{\lambda}{2}\left(\langle\operatorname{Tr}(\varepsilon)\rangle_{+}\right)^{2}+\mu \operatorname{Tr}\left\{\left(\varepsilon^{+}\right)^{2}\right\},
$$

where $\varepsilon$ is the linearized strain tensor and $\langle x\rangle_{+}=(x \pm|x|) / 2$ and $\varepsilon^{ \pm}$are compression and tensile parts of the strain tensor (see e.g. [25,28]). The choice of the numerical parameter $l$ is a central issue in the method, which is precisely discussed in the present work.

In (3), $\boldsymbol{\sigma}=\frac{\partial W}{\partial \boldsymbol{\varepsilon}}$ is the second-order Cauchy stress tensor, $\mathbf{f}$ are body forces and $\overline{\mathbf{u}}$ and $\overline{\mathbf{F}}$ are prescribed displacements and forces on the corresponding boundaries $\partial \Omega_{u}$ and $\partial \Omega_{F}$, respectively. The symbols $\nabla($.$) and \nabla \cdot($.$) denote$ gradient and divergence operators, respectively. The constitutive law is expressed (see e.g. [28]) by:

$$
\boldsymbol{\sigma}=\left((1-d)^{2}+k\right)\left\{\lambda\langle\operatorname{Tr} \varepsilon\rangle_{+} \mathbf{1}+2 \mu \varepsilon^{+}\right\}+\lambda\langle\operatorname{Tr} \varepsilon\rangle_{-} \mathbf{1}+2 \mu \varepsilon^{-}
$$

where $k$ is a small numerical parameter to avoid loss of stability in case of fully damaged elements.

Eqs. (2)-(3) are solved by a standard FE procedure in a staggered scheme at each time step (load increment). More theoretical and practical details can be found e.g. in $[25,28]$.

\section{Discussion on the influence of input parameters in the numerical simulations}

In this section, we discuss the influence of the numerical parameters on the simulation results. More specifically, we study the influence of: (a) the mesh size, (b) the loading increments size, (c) the regularization parameter $l$ in (2). For this purpose, we consider a benchmark problem with features similar to that of the experimental tests studied in the following. The benchmark described in Fig. 1 consists into a drilled sample subjected to compression. Compression tests are often preferred to tensile ones in civil engineering because of their better stability during crack propagation (see e.g. [36,39]). More details about the real corresponding experimental test are provided in the following. The geometry of the sample and boundary conditions are depicted in Fig. 1. The material parameters have been chosen as $E=12 \mathrm{GPa}, \nu=0.3$ and $g_{c}=1.4$ $\mathrm{N} / \mathrm{m}$ from the experimental values provided in [35]. Plane strain conditions are assumed. 


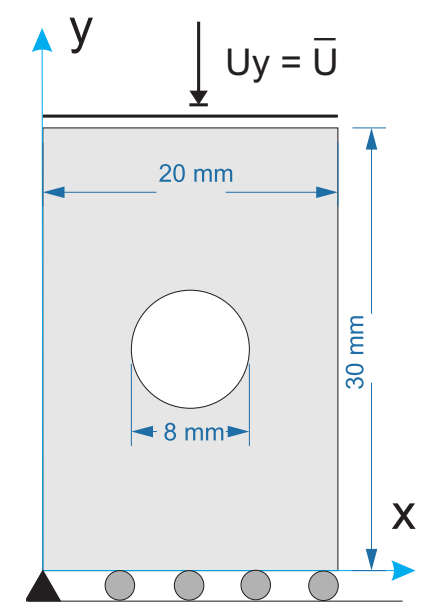

Fig. 1 Benchmark problem for analyzing the influence of numerical parameters on the simulation results: geometry and boundary conditions.

\subsection{Influence of the mesh size}

In a first test, we investigate the convergence of the mechanical response with respect to mesh refinement. In all examples of this work, linear elements have been used, i.e. triangles in $2 \mathrm{D}$ and tetrahedra in $3 \mathrm{D}$. Here, the regularization parameter is fixed to $l=0.1 \mathrm{~mm}$. We discuss in the following how to choose this parameter. It has been shown in [26] that given $l$, the criterion

$$
h \leq l / 2
$$

must be fulfilled. Monotonic compressive displacement increments of $\Delta \bar{U}=$ $-1 \times 10^{-4} \mathrm{~mm}$ have been prescribed for 250 load increments. Then we have performed several simulations using refined meshes, where the characteristic size of the elements vary between $h=0.01 \mathrm{~mm}$ and $h=0.1 \mathrm{~mm}$. Let us define the overall critical axial stress $\sigma^{*}$ as the ratio of the y-component of the resultant force prescribed at the top of the sample to the area of its upper face, when damage reaches the value $d=1$ for the first load increment at some node in the mesh (i.e. stress associated to the onset of the first crack). We study in Fig. 2 the convergence of this quantity with respect to mesh size. A clear convergence is observed, with results becoming mesh independent when condition (7) is fulfilled. This confirms the results of [26].

\subsection{Influence of the load increments}

Next, we analyze the influence of the load increment $\Delta \bar{U}$ in the numerical simulation on the mechanical response. We have used several load increments from $\Delta \bar{U}=1.5 \times 10^{-3} \mathrm{~mm}$ to $\Delta \bar{U}=3 \times 10^{-5} \mathrm{~mm}$. Results are presented in Fig. 3. In Fig. 3 (b), we study the evolution of $\sigma^{*}$ with respect to $\Delta \bar{U}$ and 


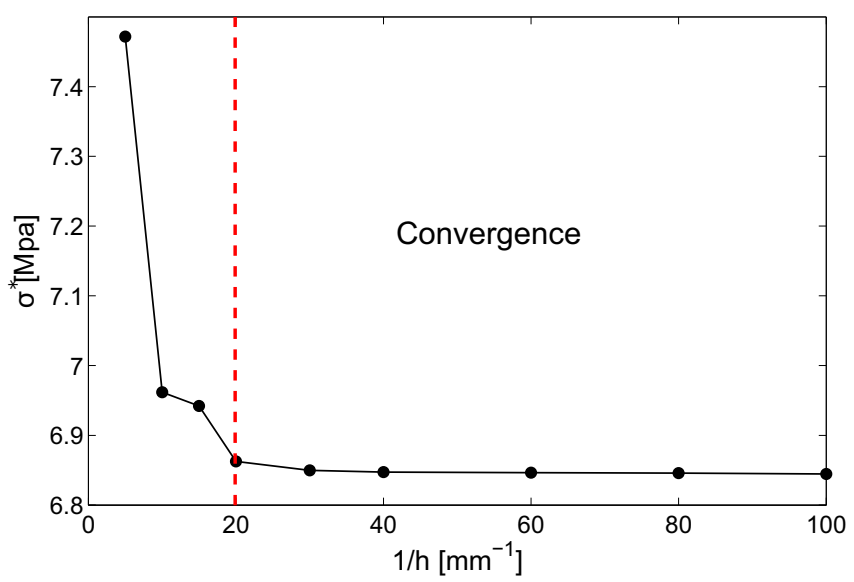

Fig. 2 Convergence of the solution with respect to mesh size.

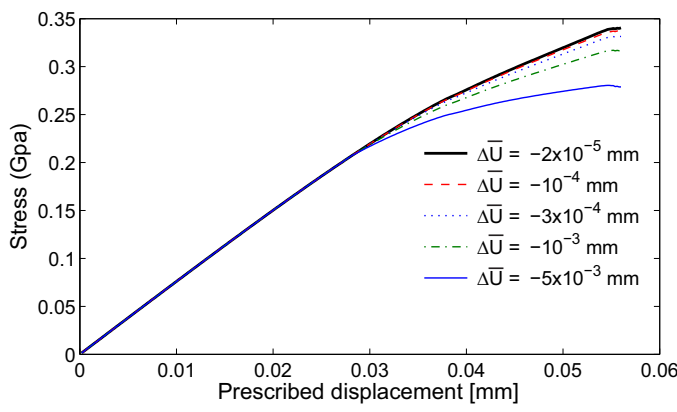

(a)

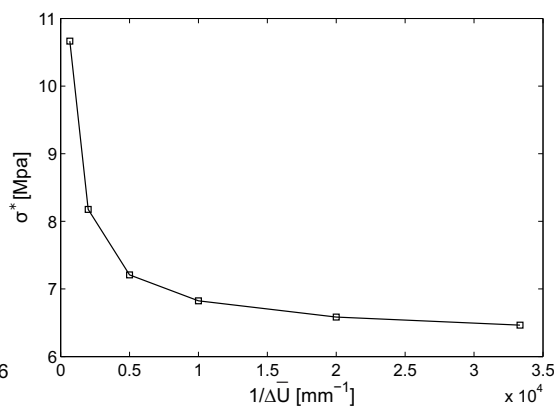

(b)

Fig. 3 Convergence of the solution with respect to the displacement increments in the numerical simulation: (a) Load - displacement curve; (b) $\sigma^{*}$ for various displacement increments.

can note the related convergence: the variation of $\sigma^{*}$ is below $2 \%$ when the increment goes from $5 \cdot 10^{-5}$ to $3 \cdot 10^{-5} \mathrm{~mm}$. This confirms the stability of the easy-to-implement staggered algorithm as soon as sufficiently small loading steps are used. Too large steps tend to delay the initiation of damage and thus harden the overall response of the structure.

\subsection{Choice of the regularization parameter $l$}

In the following, we show that the regularization parameter $l$ in (2) depends on material parameters. To illustrate this point, we consider a bar under uniaxial traction as depicted in Fig. 4. We assume that the Poisson ration is zero. In 


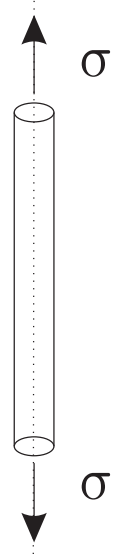

Fig. 4 1D problem for the analysis of the phase method in an initially homogeneous situation.

this configuration and in the absence of initial defects, the damage distribution is assumed to be homogeneous, i.e. $\nabla d(\mathbf{x})=0$.

Assuming an isothermal process, the reduced form of the Clausius-Duhem inequality can be written as:

$$
\mathcal{A} \dot{d} \geq 0
$$

where

$$
\mathcal{A}=-\frac{\partial E}{\partial d}
$$

is the thermodynamic force associated with $d$ and $\dot{d}$ denotes derivative with respect to time $t$. We assume that the evolution of the damage parameter $d$ is governed by the simple negative threshold function

$$
F(\mathcal{A})=\mathcal{A} \leq 0
$$

such that when $F(\mathcal{A})<0$ no evolution of damage occurs. The principle of maximum dissipation requires the dissipation $\mathcal{A} \dot{d}$ to be maximum under the constraint (10). By using the method of Lagrange multipliers, we define the Lagrangian as:

$$
\mathcal{L}=-\mathcal{A} \dot{d}+\lambda F(\mathcal{A})
$$

where $\lambda$ is the Lagrange multiplier associated with the constraint (10). Minimizing $\mathcal{L}$ under the constraint (10) yields the Kuhn-Tucker equalities:

$$
\frac{\partial \mathcal{L}}{\partial \mathcal{A}}=0, \quad \lambda \geq 0, \quad F \leq 0, \quad \lambda F=0 .
$$


The left-hand equation in (12) gives $\lambda=\dot{d}$. Then for $\dot{d}>0, F(\mathcal{A})=0=$ $\mathcal{A}=-\frac{\partial E}{\partial d}$, which leads to (see more details in $[28]$ ):

$$
F=2(1-d) \Psi^{+}-g_{c} \delta \gamma(d)=0 .
$$

In $(13), \delta \gamma(d)$ is given by $\delta \gamma=\frac{d}{l}-l \Delta d[25]$, where $\Delta d$ is the Laplacian of $d$. For a uniform damage parameter as in the considered 1D problem, $\delta \gamma$ reduces to $\delta \gamma=d / l$.

For uniaxial tension, and assuming $k \simeq 0$ we can write from (6):

$$
\sigma=g(d) E \varepsilon, \quad \Psi^{+}=\frac{1}{2} E \varepsilon^{2},
$$

with $g(d)=(1-d)^{2}$. Then using (13), we obtain the relation:

$$
(1-d) E \varepsilon^{2}-\frac{g_{c}}{l} d=0 .
$$

The strain and stress can then be expressed by:

$$
\begin{aligned}
& \varepsilon(d, l)=\sqrt{\frac{g_{c} d}{l E(1-d)}}, \\
& \sigma(d, l)=\sqrt{d(1-d)^{3}} \sqrt{\frac{E g_{c}}{l}} .
\end{aligned}
$$

The maximum value of the stress with respect to $d$ is given by:

$$
\sigma_{c}=\operatorname{Arg}\left\{\sup _{\mathrm{d}=\left[\begin{array}{ll}
0 & 1
\end{array}\right]} \sigma(\mathrm{d}, \mathrm{l})\right\}
$$

which is reached for $d=1 / 4$, corresponding to the critical value of the stress $\sigma_{c}$ :

$$
\sigma_{c}=\frac{9}{16} \sqrt{\frac{E g_{c}}{3 l}}
$$

and of the strain:

$$
\varepsilon_{c}=\sqrt{\frac{g_{c}}{3 l E}}
$$

These obtained formulations are similar with the result in the work of Amor et al [1]. Analyses leading to similar relationships can also be found in $[19,5$, $7,31]$. From these expressions, it is clear that the critical stress will increase as $l$ decreases. In the limit of $l$ tending to zero, i.e., when the phase-field formulation coincides with the discrete fracture formulation, the crack nucleation stress becomes infinite. This observation is consistent with the predictions of Griffith's theory, which only allows for crack nucleation at stress singularities. Eq. (20) gives a relationship between $l$ and the material parameters, namely the Young modulus, $E$, the Griffith critical surface energy, $g_{c}$, and a value of the tensile strength $\sigma_{c}$ determined experimentally, and denoted in that case 


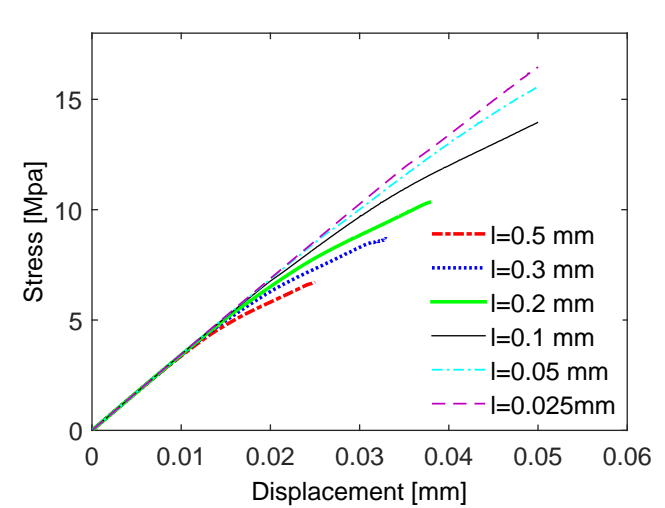

(a)

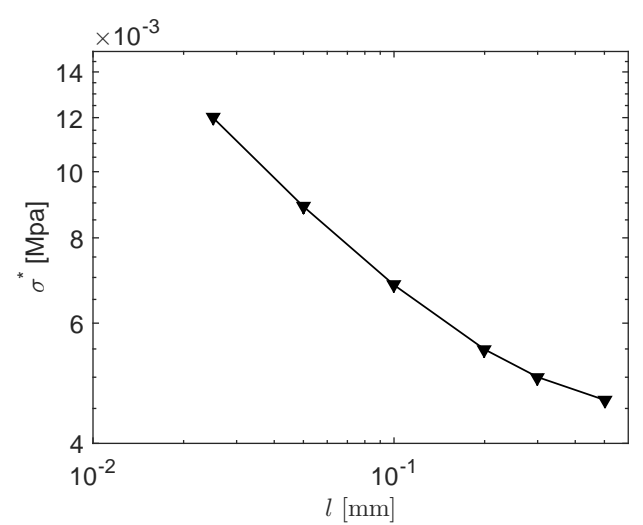

(b)

Fig. 5 Evolution of the solution with respect to the regularization parameter $l$ : (a) Load displacement curve; (b) $\sigma^{*}$ versus $l$.

by $\sigma_{c}^{e x p}$, which now refers to the critical stress leading to rupture in a uniaxial uniform tension test:

$$
l=\frac{27 E g_{c}}{256\left(\sigma_{c}^{e x p}\right)^{2}} .
$$

Note that this relation holds for uniaxial traction without damage gradient and only provides an estimation for $l$ but clearly shows that $l$ can be linked to material parameters. From the values of $g_{c}$ and $\sigma_{c}^{e x p}$ identified experimentally in [35] for a plaster material, i.e. $E=12 \mathrm{GPa}, \sigma_{c}=3.9 \mathrm{MPa}$ and $g_{c}=1.4$ $\mathrm{N} / \mathrm{m}$ we obtain $l \simeq 0.1 \mathrm{~mm}$.

In the next test, we show numerically that the mechanical response does not converge with respect to the parameter $l$. An unstructured mesh with minimal element size $h_{\min }=0.01 \mathrm{~mm}$ is employed around the hole where the cracks should initiate, and with maximal element size $h_{\max }=1 \mathrm{~mm}$ away from the hole, such that mesh size ensures numerical convergence of the computations for all values of $l$ considered hereafter. The displacement increment is chosen as $\Delta \bar{U}=10^{-4} \mathrm{~mm}$. In Fig. 5 (a), the evolution of the solution with respect to the regularization parameter $l$ is plotted for different values of $l$ ranging from $0.025 \mathrm{~mm}$ to $0.5 \mathrm{~mm}$. In Fig. 5 (b), the stress required to onset the first crack $\sigma^{*}$ is plotted versus $l$. While the force-displacement curve in Fig. 5 (a) seems to converge when $l$ decreases (indeed towards a purely elastic response), it is obvious that this is not the case for the value of $\sigma^{*}$. This test illustrates the fact that the regularization parameter $l$ must be identified as a material parameter, i.e. each value of $l$ will lead to a different response of the structure. 


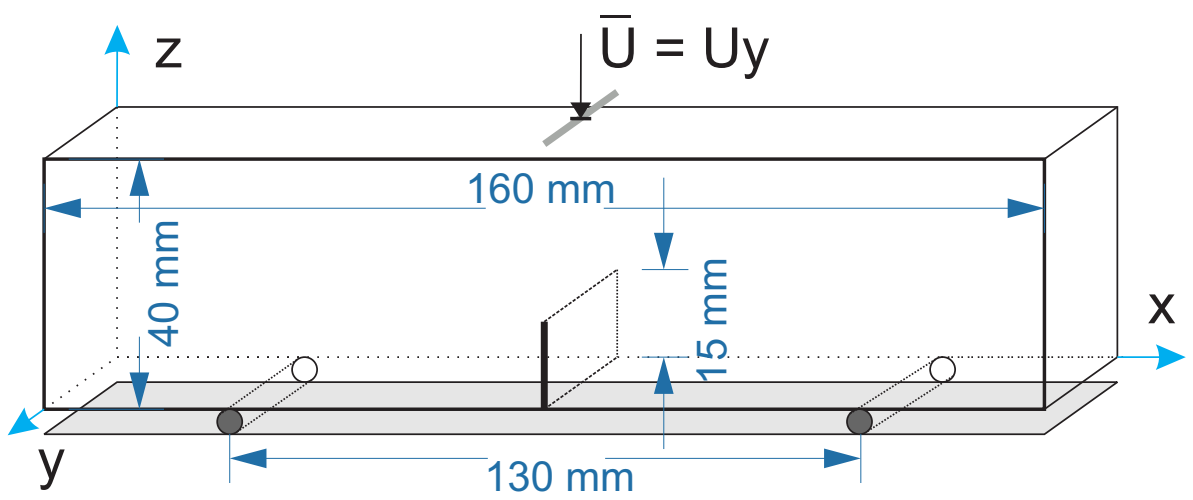

Fig. 6 3D 3-point bending test: Geometry and boundary conditions.

\section{Experimental validation: three-point bending test}

\subsection{Pre-notched beam}

In this test, we validate the phase field solution on an experimental 3-point bending test of a beam containing an initial crack of length $15 \mathrm{~mm}$. The geometry, dimensions, and boundary conditions of the structure are depicted in Fig. 6. The material is dry plaster, composed of plaster powder of the Siniat Company named Prestia Profilia $35 \AA$. The plaster sample preparation are detailed in a previous work [35]. In the mentioned study, the material parameters have been identified experimentally and are the same as in the previous example: $E=12 \mathrm{GPa}, \nu=0.3, g_{c}=1.4 \mathrm{~N} / \mathrm{m}$ and $\sigma_{c}^{e x p}=3.9 \mathrm{MPa}$, which give the value of $l=0.1 \mathrm{~mm}$ from (21). Note that here the Poission ratio is non zero and the problem is not one-dimensional, thus (21) only provides an estimation for $l$. More crucially, the derivation of (21) does only hold for structures with homogeneous displacements and fracture fields before fracture nucleation.

The $z$-component of displacements $\bar{U}$ is prescribed along a line in the middle of the upper face, while the all components of displacements are blocked along two lines on the lower face (see Fig. 6).

Three-dimensional simulations have been conducted. A refined mesh was constructed using tetrahedral elements, with $h_{\max }=3 \mathrm{~mm}$ and $h_{\min }=0.05$ $\mathrm{mm}$ in the region of expected crack path, to satisfy the condition $h_{\min } \leq l / 2$. Monotonic compressive displacement increments of $\Delta \bar{U}=-5 \times 10^{-4} \mathrm{~mm}$ have been prescribed as long as $d<0.9$ in all elements and $\Delta \bar{U}=-5 \times 10^{-5} \mathrm{~mm}$ as soon as $d>0.9$ in one integration point. The crack propagation evolution is depicted in Fig. 7 for two loading stages.

Fig. 8 provides the load-displacement curve obtained from the simulation. The critical load $F_{r}$ is defined as the maximum resultant load before softening due to crack propagation. We compare this critical load with the experimental 


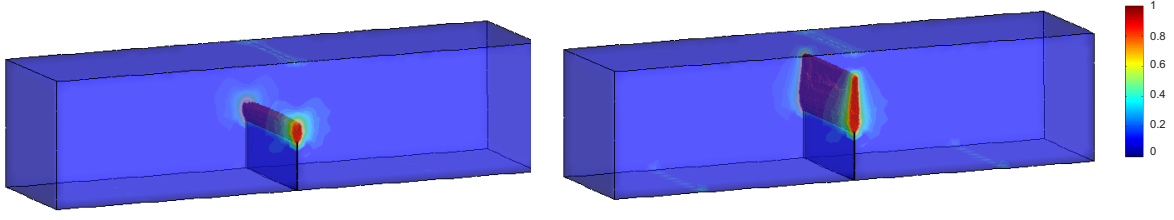

Fig. 7 3-point bending test, crack evolution (damage variable $d(\mathbf{x})$ ) for two prescribed displacements: $\bar{U}=0.15 \mathrm{~mm}$ and $\bar{U}=0.18 \mathrm{~mm}$.

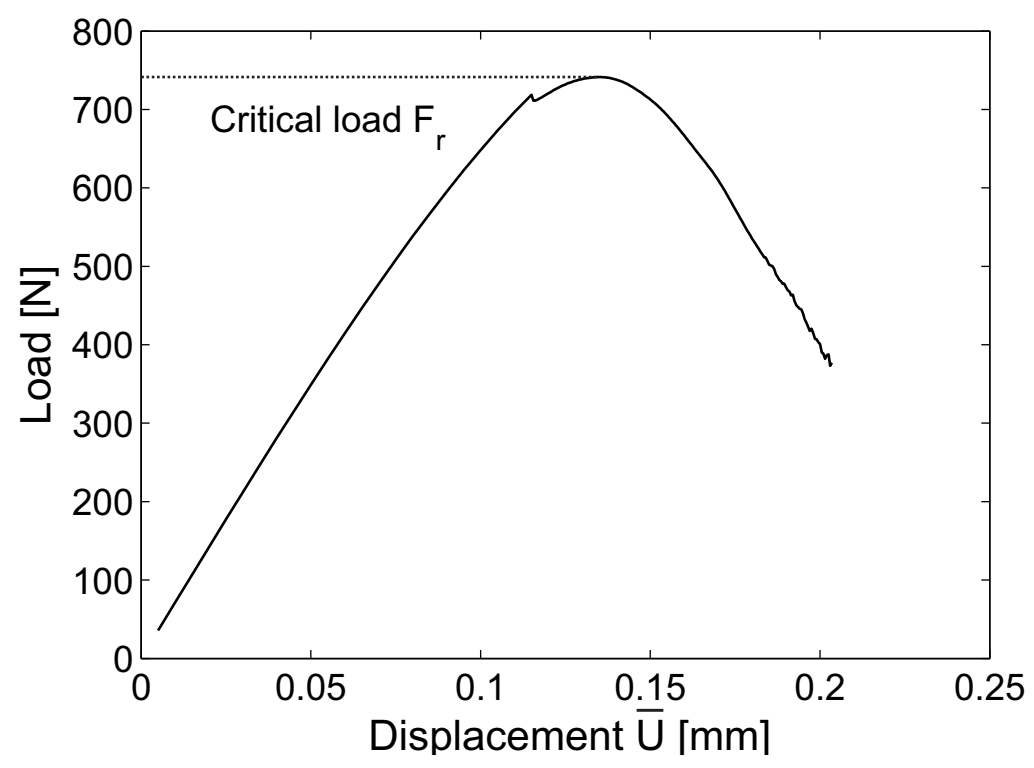

Fig. 8 Load - displacement curve for the 3-point bending test: numerical model.

values provided in [35] for several samples in Fig. 9 and can note that we obtain a good agreement for the values of $F_{r}$ with respect to experiments.

\subsection{Un-notched beam}

We investigate now the capability of the phase field method to provide a correct estimated value of $\sigma_{c}^{e x p}$ for crack initiation in a structure different from the one in which the critical stress $\sigma_{c}^{n u m}$ was identified. For this purpose, we consider an un-cracked beam under three-point bending, as depicted in Fig. 10.

The stress is evaluated numerically during the simulation in an element located on the known path of the crack. The tensile strength $\sigma_{c}^{\text {num }}$ is defined as the maximal stress evaluated numerically before softening in the integration point of an element located in the middle of the lower end, as depicted in Fig. 11. In the present work, we have used linear finite elements, with one Gauss integration point per element. From now on and in all following examples, all 


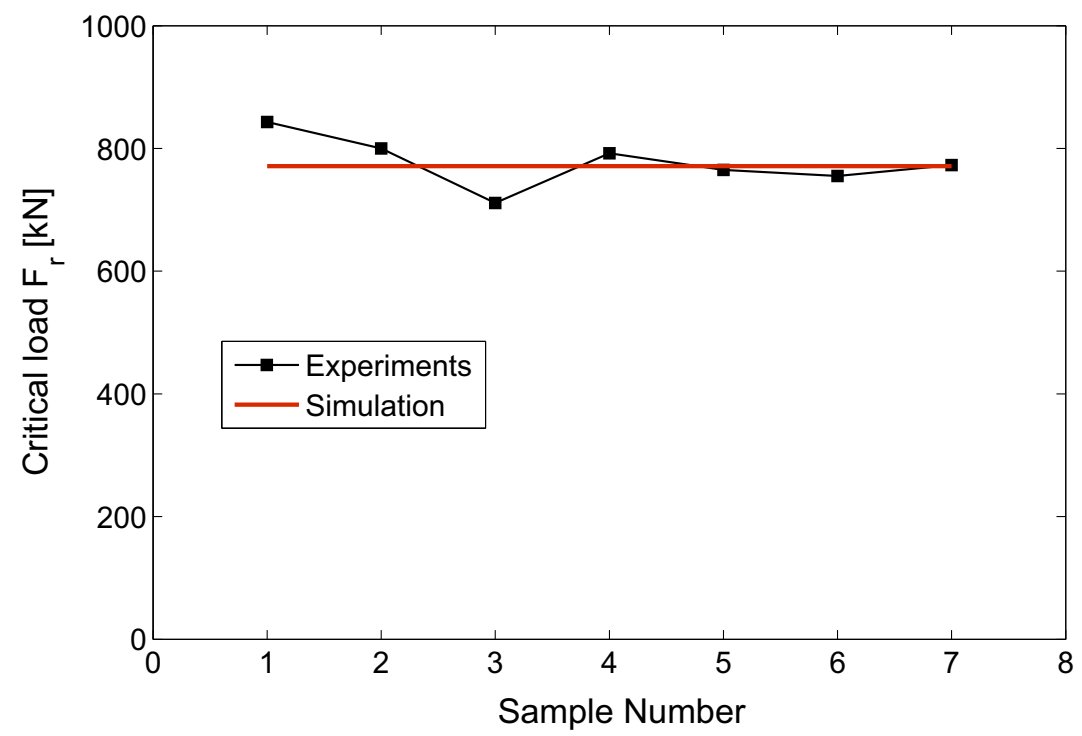

Fig. 9 Critical load for the 3-point bending problem: comparison between experiments and numerical predictions.

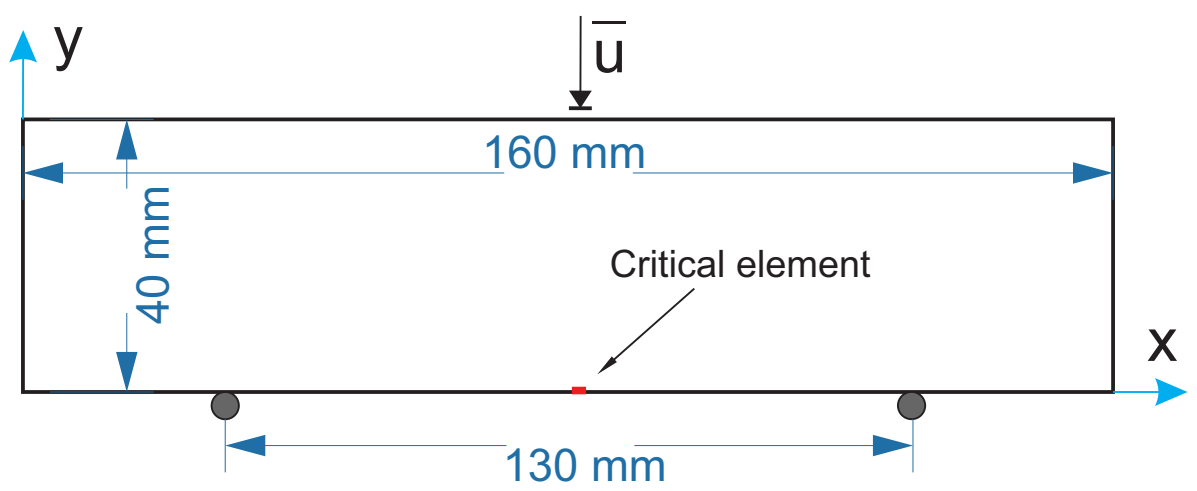

Fig. 10 2D 3-point bending test of non cracked beam: Geometry and boundary conditions.

material parameters are the same as in the previous example and $l$ is equal to $0.1 \mathrm{~mm}$. Monotonic compressive displacement increments of $\Delta \bar{U}=-2 \times 10^{-3}$ $\mathrm{mm}$ have been used for 180 increments. We obtain a good agreement between the value predicted numerically $\left(\sigma_{c}^{\text {num }}=4.01 \mathrm{MPa}\right)$ and the experimental value identified from another experiment in [35] $\left(\sigma_{c}^{e x p}=3.9 \mathrm{MPa}\right)$. 


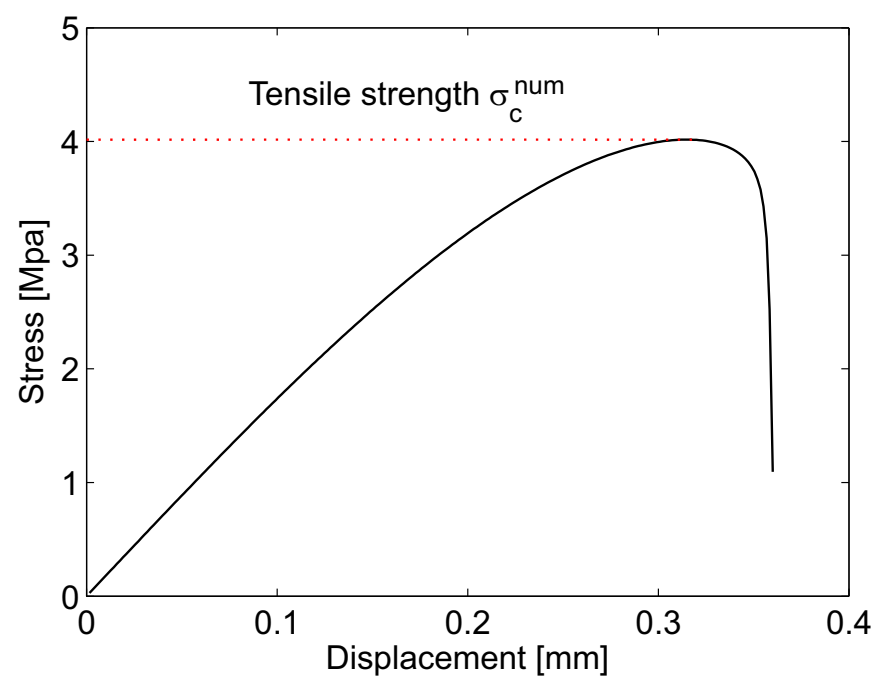

Fig. 11 Tensile strength for the 3-point bending problem: stress - displacement curve of the critical element

\section{Experimental validation: compression of a drilled plaster specimen containing a single cylindrical hole}

In the following, we investigate crack initiation and propagation in a more involved test, and compare the numerical prediction with experimental results provided in [35]. The objective is to evaluate if the numerical model is able to predict accurately the response of cracked structure in other configurations than the ones used to identify the material parameters.

A drilled sample is considered, as depicted in Figure 12. A thick plate contains one single cylindrical hole with diameter $D$. Several samples with various hole diameters, ranging from $D=3$ to $D=6 \mathrm{~mm}$, have been tested. The dimensions of the plate are $100 \mathrm{~mm} \times 65 \mathrm{~mm} \times 40 \mathrm{~mm}$. The material (plaster) is the same as in the previous example. The sample is loaded in compression. In the experimental tests, the load is applied continuously at a speed of $0.2 \mathrm{~mm} / \mathrm{min}$. Consistently, the numerical calculations are run in the quasi static regime, as for previous cases. PMMA plates were used on top and bottom face to reduce the lack of planarity, parallelism and friction conditions [34] to avoid stress concentration.

Experimental image correlation data were provided in [35], together with force measurement to detect the crack experimentally. A high-resolution camera (Baumer HXC20, progressive scan sensor with $2048 \times 1088$ pixels), with a pixel size of $5.5 \times 5.5 \mu \mathrm{m}^{2}$, and equipped with a ZEISS Makro-Planar $100 \mathrm{~mm}$ macro lens was used to continuously acquire images of the specimen during loading at a frame rate of $20 \mathrm{~Hz}$. As the detection of the crack onset is not possible with naked eye, the recorded images were processed by $2 \mathrm{D}$ digital im- 


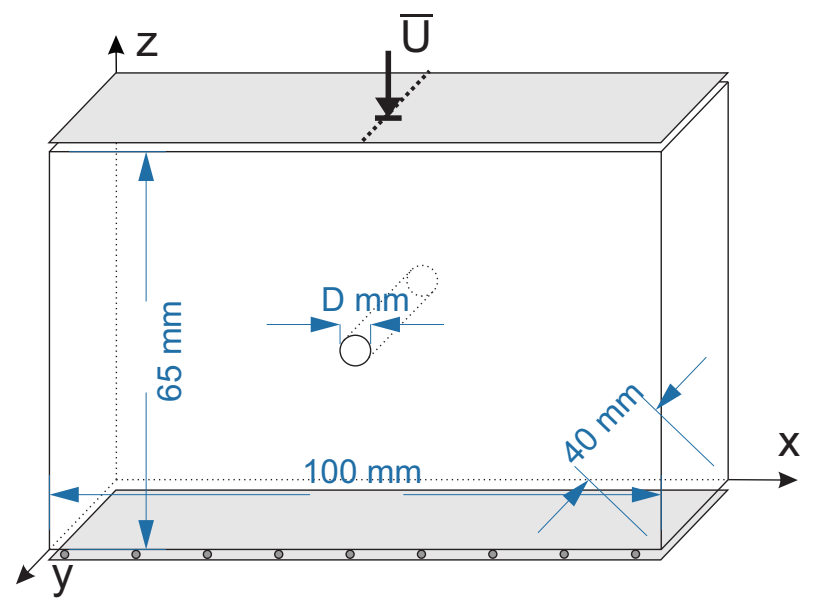

Fig. 12 Plaster sample containing one cylindrical drilled hole: geometry and boundary conditions for both experimental setup and simulation.

age correlation (DIC) techniques. Cracks are detected by high levels of local $x x$-strain components, measured for a gage length defined by the mesh of correlation points (20 pixels spacing), which is the signature of the presence of displacement discontinuities between two points of the mesh.

The 2D technique of digital image correlation 2D-DIC was used. When the sample is subjected to a compressive load, two opposite cracks initiate on top and bottom of the hole and grow from the cavity, in a direction parallel to the load. In [35], the experimental results have been compared to the semi-analytical model of Leguillon [21], which requires numerical FEM computations to evaluate the stress intensity factors. In the mentioned work, 2D FE simulations with plane strain assumptions were used. In view of the dimensions of the sample and owing to the fact the measurements are performed at the surface of the sample, the plane strain assumption might be questionable. For this purpose, we have performed 2D simulations with both plane strain and plane stress assumption, as well as full 3D simulations. The boundary conditions model the experimental ones on the sample, and are described for the $3 \mathrm{D}$ case in Fig. 12: on the lower surface $(z=0)$, the $z$ - displacements are fixed and the $x$ - and $y$ - displacements are free. On the upper end, the $x-$ and $y-$ displacements are free, while the $z$ - displacements are prescribed, with an increasing value $\bar{U}$ during the simulation. Monotonic compressive displacement increments of $\Delta \bar{U}=-10^{-3} \mathrm{~mm}$ are prescribed for first load increments and as soon as $d$ reaches 0.9 in one integration point of the Finite Element mesh, we use $\Delta \bar{U}=-10^{-4}$. A finite element mesh with varying element size $\left(\mathrm{h}_{\min }=\right.$ $0.05 \mathrm{~mm}$ around the hole and $\mathrm{h}_{\max }=0.25 \mathrm{~mm}$ in the rest of domain) is used.

In Figs. 13 -14, we show a comparison of the experimental digital image correlation technique used to detect the crack evolution and the simulation, were the damage field, associated with the crack, is depicted. This case corresponds to a diameter $D=5 \mathrm{~mm}$. We can note that the numerical solution 


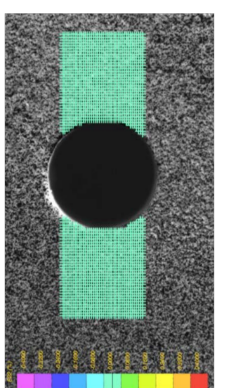

(a)

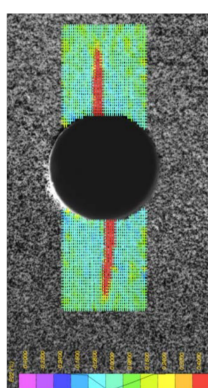

(b)

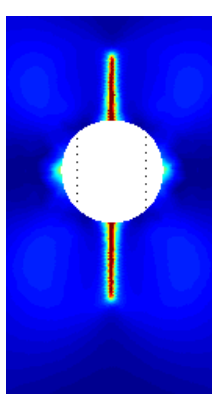

(c)

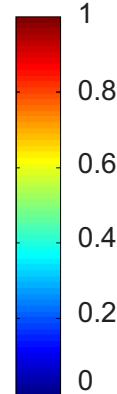

0

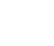

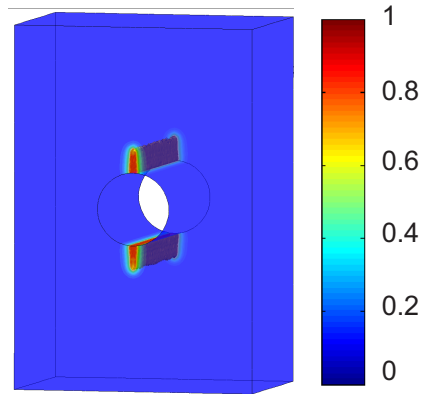

(d)

Fig. 13 Crack path evolution near the cylindrical hole $(D=5 \mathrm{~mm})$; (a) and (b): Strain maps obtained with digital image correlation for initial and loaded state [35], for 15.2 MPa and 14.1 MPa, respectively; (c) 2D simulation (plane strain); (d) 3D simulation (damage variable $d(\mathbf{x}))$.

based on the phase field method can capture the crack initiation on top and bottom of the hole and the vertical path of the two cracks. In addition, the length of the crack for the given load is accurately predicted (Figs. 13 (b) and (c)).

In the simulations, the crack length is computed as the distance between the last point for which $d=1$ and the hole boundary, assuming a straight crack. The same procedure is employed in 3D. In Fig. 15 we quantitatively compare the crack length evolution with respect to the applied load computed at the point where the displacement is prescribed. Results for $2 \mathrm{D}$ plane strain and plane stress, 3D simulations and experimental DIC results are compared in Fig. 14. Fig. 15 shows that all three models provide a satisfying prediction for the critical load corresponding to the onset of the crack. However, we can note that during propagation, the experimental evolution deviates from $2 \mathrm{D}$ predictions. The $3 \mathrm{D}$ simulation is in that case in better agreement with the experimental response for both top and bottom cracks.

To analyze the influence of the diameter of the hole on the stress at the time cracks onset, several samples with diameters varying between 3 and $6 \mathrm{~mm}$ have been prepared and tested. Simulations have been performed here also in $2 \mathrm{D}$ and $3 \mathrm{D}$. Results are provided in Figure 16. They show the good ability of the simulation model to accurately predict the evolution of critical load $\sigma^{*}$ (onset of the crack) with hole diameters and related size effects. The ability of the phase field method to reproduce size effects has also been discussed in [18]. A general assessment of different models in this context can be found in [22], where the authors find that 2 parameter fracture models can model size effects only to a certain extend. 


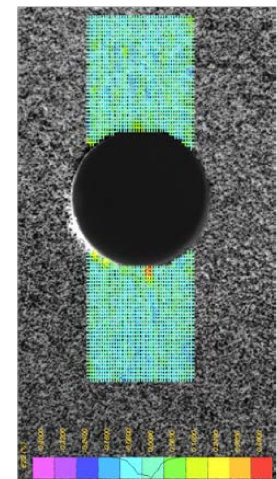

7.84 MPa

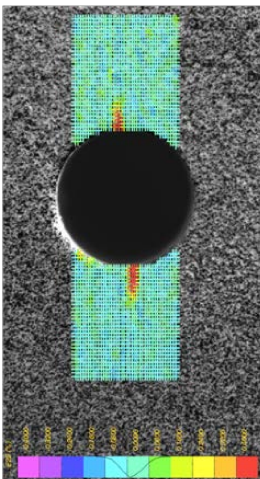

8.51 MPa

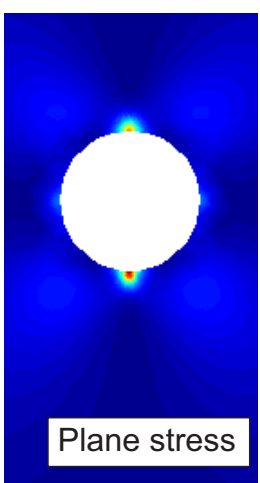

$7.89 \mathrm{MPa}$

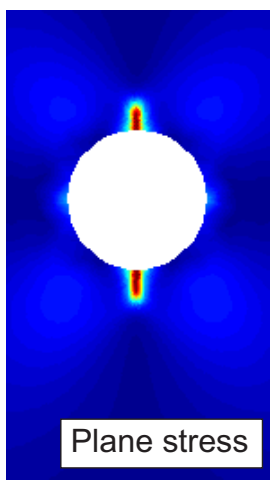

8.57 MPa

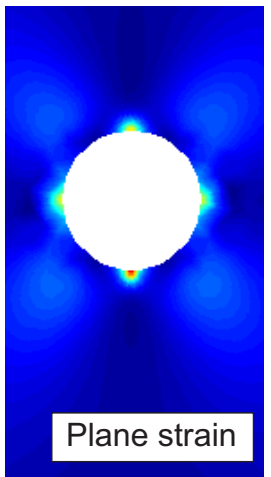

7.81 MPa

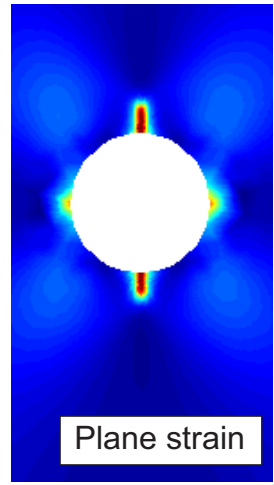

8.47 MPa

Fig. 14 Crack propagation at different values of the applied stress around the cylindrical hole: comparison between experiments (digital image correlation) and simulations (damage variable $d(\mathbf{x}))$.

6 Microcracking in a plaster specimen containing a periodic distribution of cylindrical holes

In this last example, we investigate the microcracking of two plaster specimens containing many holes, whose configurations are depicted in Fig. 17. In both cases, the diameter of the holes is $D=4 \mathrm{~mm}$. Configuration of Fig. 17 (a) corresponds to a volume fraction of $12.2 \%$, and in Fig. 17 (b) to $13.5 \%$. A FE adaptive mesh with characteristic size $h_{\min }=0.05 \mathrm{~mm}$ has been used around the holes, and larger elements whose size are $h_{\max }=0.5 \mathrm{~mm}$ have been employed away from holes. The whole mesh contains 905437 elements.

$2 \mathrm{D}$ plane strain simulation was conducted. Monotonic compressive displacement increments are prescribed on the top edge of the specimen, with $\Delta \bar{U}=-10^{-3} \mathrm{~mm}$ in the first 1000 increments and $\Delta \bar{U}=-5 \times 10^{-5} \mathrm{~mm}$ in the last 1500 increments. The evolution of microcracking within the specimen 


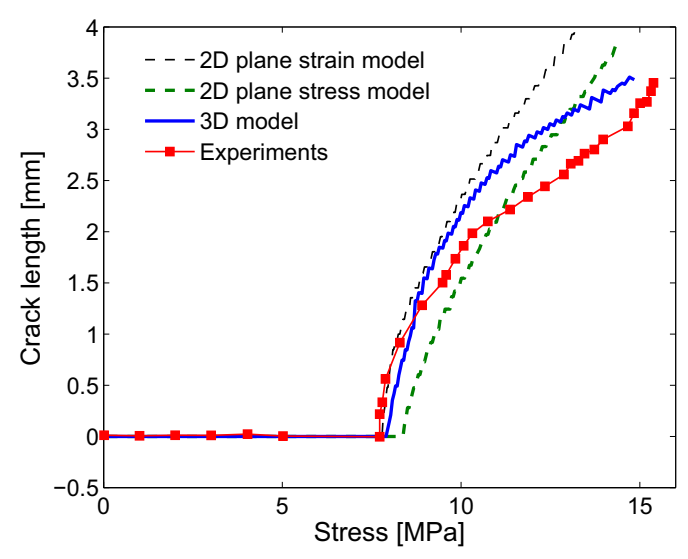

(a)

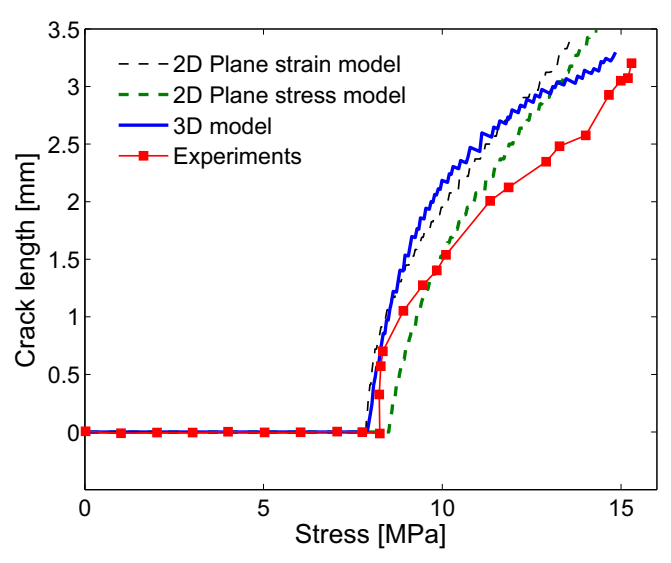

(b)

Fig. 15 Evolution of the crack length with respect to the resultant stress on the upper boundary, comparison between models and experimental data: (a) top crack; (b) bottom crack.

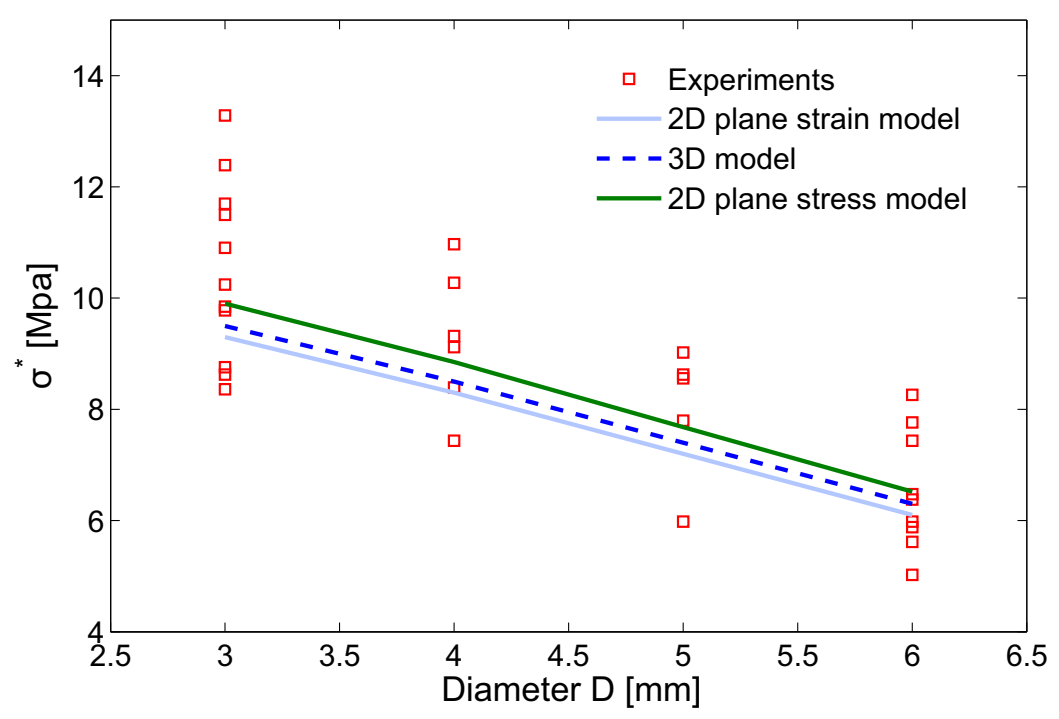

Fig. 16 Stress associated with the cracks onset with respect to the cylindrical hole diameter: comparison between experiments and numerical simulation.

is depicted in Fig. 18. The simulation model captures well the vertical propagation of the different microcracks. The microcracks propagate faster near the left and right boundaries than in the central region, probably because of the influence of the free boundary conditions on the lateral surfaces. In addition, there is also a slight dissymmetry between upper and lower parts of the 


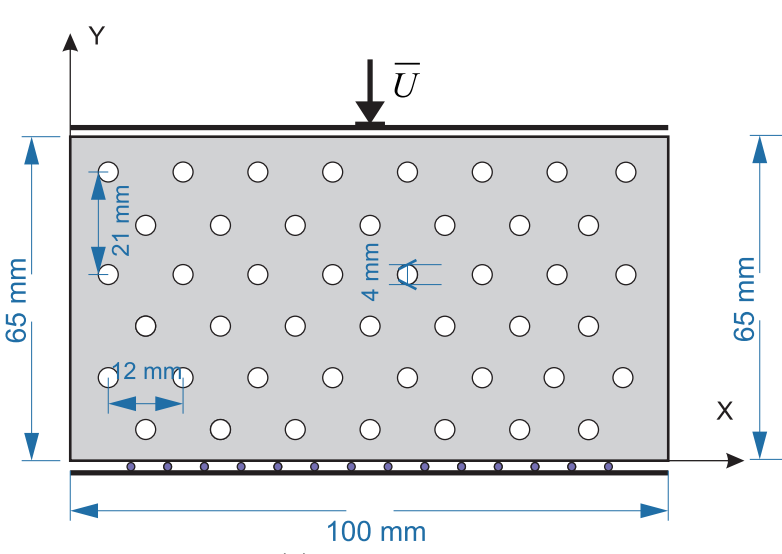

(a)

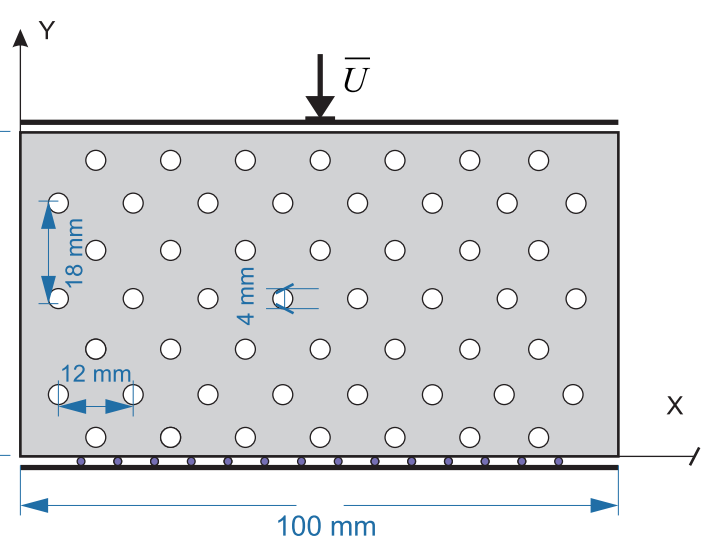

(b)

Fig. 17 Plaster specimen containing a regular distribution of cylindrical holes: (a) surface fraction $12.2 \%$ and (b) surface fraction $13.5 \%$ [34].

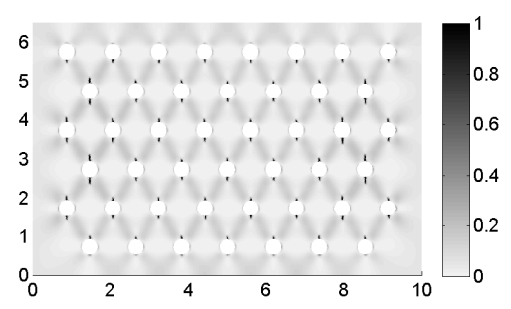

(a)

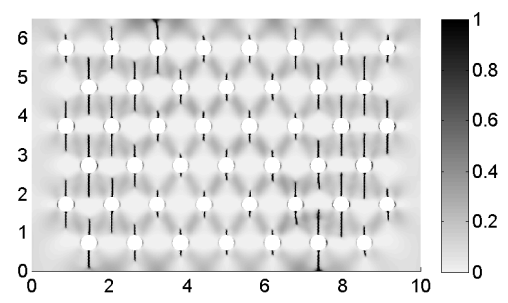

(c)

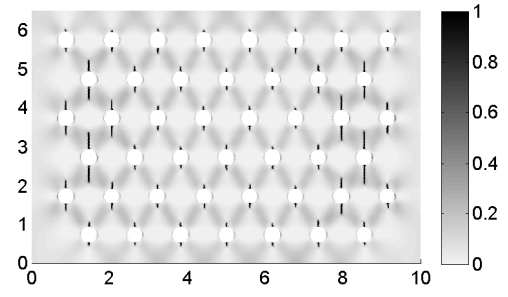

(b)

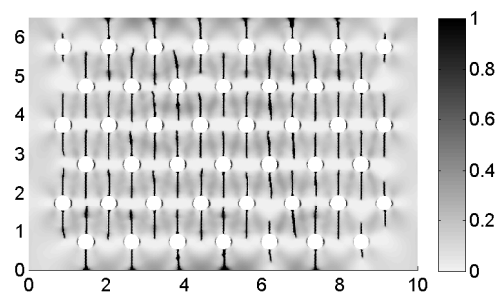

(d)

Fig. 18 Plaster specimen containing regular distribution of cylindrical holes: evolution of the microcracking for different compressive loads (damage variable $d(\mathbf{x})$ ): (a) $\bar{U}=0.544$ $\mathrm{mm}$; (b) $\bar{U}=0.594 \mathrm{~mm}$; (c) $\bar{U}=0.64 \mathrm{~mm}$; (d) $\bar{U}=0.67 \mathrm{~mm}$;

sample (a), whose successive damage maps are reproduced in figure 18. This is linked to the absence of horizontal symmetry for this sample. For sample (b), the hole distribution is symmetric between upper and lower parts, and the resulting simulated damage map is also symmetric. 


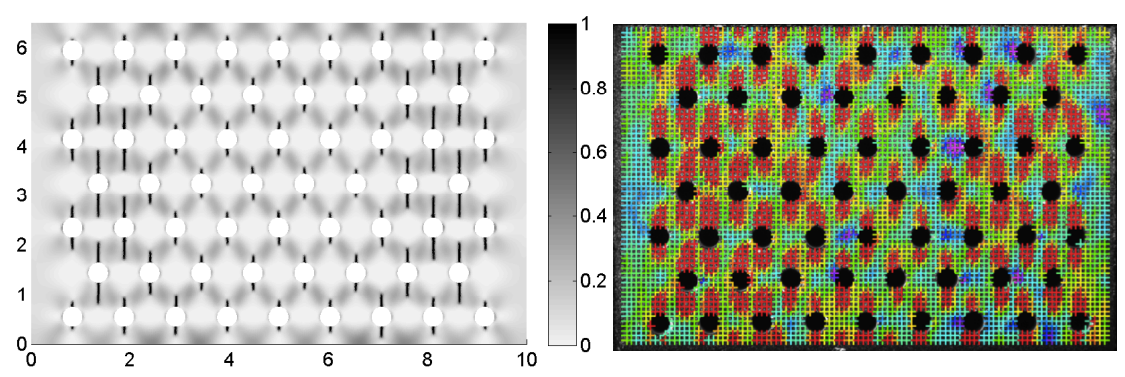

(a)

(b)

Fig. 19 Crack trajectory comparison between the present simulation (a) and the experiment (b) provided in [34] (damage variable $d(\mathbf{x})$ ) for $\bar{U}=0.614 \mathrm{~mm}$.

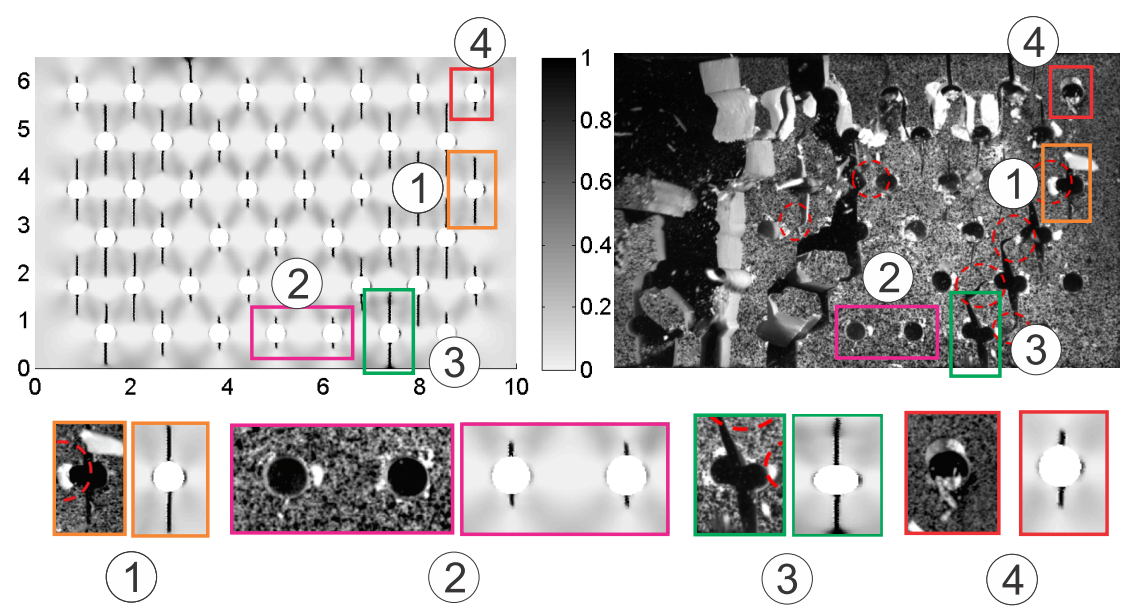

Fig. 20 Qualitative comparison of the microcracking propagation between the present simulation and the experiment provided in [34] (damage variable $d(\mathbf{x})$ ) for $\bar{U}=0.64 \mathrm{~mm}$.

In Fig. 19 the microcracking pattern for the case of porous fraction $13.5 \%$ is depicted and numerical simulations and digital correlation image obtained in [34] are qualitatively compared. Globally speaking, the heterogeneity of the damage map between central and lateral parts of the sample is nicely captured by the computation. In Fig. 20, we compare with more details the microcracking morphology between the simulation and the experiment provided in [34], [35], and note that it is qualitatively captured, both regarding the vertical propagation of the different cracks, and regarding the non uniform propagation of the microcracks within the sample.

To compare more quantitatively the predictions provided by the numerical simulation, we analyze the effects of changing the configuration (volume fraction and distribution) with respect to the stress required to initiate the first cracks in the sample and to generate cracks around all holes. Again, the corresponding experimental values have been provided in [34]. Comparisons are 


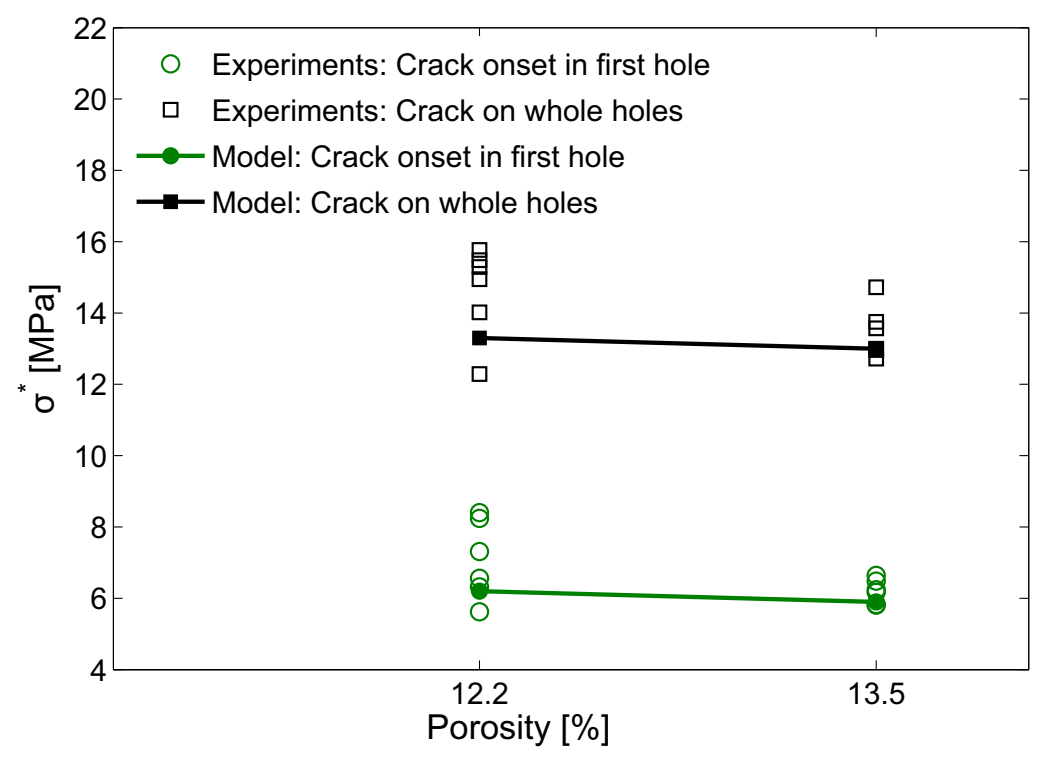

Fig. 21 Stress corresponding to crack onset within the specimen: comparison between experimental results and the numerical model for two porosities.

provided in Fig. 21. The numerical simulation method is in good agreement with the experimental values.

\section{Conclusion}

In this work, we have discussed the choice of the parameters in the phase field method, which is a promising simulation tool for initiation and propagation of cracks in brittle materials. More specifically, we have analyzed the influence of the numerical parameters and have validated the fact that the regularization parameter describing the width of the smeared crack approximation is linked to material parameters. The regularization length then requires experimental measures to be identified. We have shown that the other numerical parameters (load increments, mesh size) lead to convergent responses when they decrease. Then, from the knowledge of the elastic parameters, of the fracture resistance and of the regularization parameter of the phase field method, essentially identified from experimental measurements of critical stress in uniformly stressed samples, we have conducted several simulations, including crack initiation and propagation in three-point bending beam and in drilled samples of plaster in compression. Remarkably, the phase field model is able to predict quantitatively crack paths, crack propagation morphologies, and mechanical response with good agreement regarding experimental results for other geometrical configurations than the ones used to identify the material parameters. Thus, the 
phase field method constitutes a promising tool for prediction of strength in brittle heterogeneous or lightweight materials for civil engineering.

Acknowledgements This work has benefited from a French government grant managed by ANR within the frame of the national program Investments for the Future ANR-11-LABX022-01. The financial support of J. Yvonnet from IUF (Institut Universitaire de France) is gratefully acknowledged. We thank the support from the Federeation Francilienne de Mecanique to conduct the experimental program.

\section{Aknowledgement}

\section{References}

1. H. Amor, J.J. Marigo, and C. Maurini. Regularized formulation of the variational brittle fracture with unilateral contact: Numerical experiments. J. Mech. Phys. Solids, 57(8):1209-1229, 2009

2. Z. Bažant and T. Belytschko. Wave propagation in strain-softening bar: exact solution. journal of engineering mechanics. J. Eng. Mech., 111:81-389, 1985.

3. Z. Bažant and G. Pijaudier-Cabot. Nonlocal continuum damage, localization instability and convergence. J. Appl. Mech., 55:521-539, 1988.

4. T. Belytschko and T. Black. Elastic crack growth in finite elements with minimal remeshing. Int. J. Numer. Meth. Eng, 45:601-620, 1999.

5. A. Benalla and J.-J. Marigo. Bifurcation and stability issues in gradient theories with softening. Model. Simul. Mater. Sc., 15(1):S283-S295, 2007.

6. P.E. Bernard, N. Moës, and N. Chevaugeon. Damage growth modeling using the thick level set (TLS) approach: Efficient discretization for quasi-static loadings. Comput. Meth. Appl. Mech. Eng., 233:11-27, 2012.

7. M.J. Borden, C.V. Verhoosel, M.A. Scott, T.J.R. Hughes, and C.M. Landis. A phasefield description of dynamic brittle fracture. Comput. Meth. Appl. Mech. Eng., 217:7795, 2012.

8. R. De Borst, 1.J. Sluys, and H.B. Muhlausanf J. Pamin. Fundamental issues in finite element analysis of localization of deformation. Eng. Comput., 10:99-121, 1993.

9. B. Bourdin. Numerical implementation of the variational formulation for quasi-static brittle fracture. Interface Free Bound., 9(3):411-430, 2007.

10. G. Camacho and M. Ortiz. Computational modelling of impact damage in brittle materials. Int. J. Solids Struct., 33:2899-2938, 1996.

11. F. Cazes and N. Moës. Comparison of a phase-field model and of a thick level set model for brittle and quasi-brittle fracture. Int. J. Numer. Methods Eng., In press, 2015.

12. C. Daux, N. Moës, J. Dolbow, and T. Belytschko. Arbitrary branched and intersecting cracks with the extended finite element method. Int. J. Num. Meth. Eng., 48:1741-1760, 2000 .

13. L.O. Eastgate, JP. Sethna, M. Rauscher, and T. Cretegny. Fracture in mode i using a conserved phase-field model. Phys. Rev. E, 65(3):036117, 2002.

14. G.A. Francfort and J.J. Marigo. Revisiting brittle fracture as an energy minimization problem. J. Mech. Phys. Solids, 46(8):1319-1342, 1998.

15. V. Hakim and A. Karma. A continuum phase field model for fracture. J. Mech. Phys. Solids, 15(2):342-368, 2009.

16. M. Hofacker and C. Miehe. A phase field model of dynamic fracture: Robust field updates for the analysis of complex crack patterns. Int. J. Numer. Meth. Eng, 93(3):276301, 2013.

17. C. Kuhn and R. Müller. A continuum phase field model for fracture. Eng. Frac. Mech., 77(18):3625-3634, 2010.

18. C. Kuhn and R. Müller. Simulation of size effects by a phase field model for fracture. Theor. Appl. Mech. Lett., (5), 2014. 
19. C. Kuhn, A. Schlueter, and R. Müller. A phase-field description of dynamic brittle fracture. Comput. Mater. Sc., 108:374-384, 2015.

20. D. Lasry and T. Belytschko. Localization limiters in transient problems. Int. J. Solids Struct., 24:581-597, 1988.

21. D. Leguillon. Strength or toughness? a criterion for crack onset at a notch. Eur. J. Mech. A-Solid, 21(1):61-72, 2002.

22. J. Li and X.B. Zhang. A criterion study for non-singular stress concentrations in brittle or quasi-brittle materials. Eng. Frac. Mech., (4), 2006.

23. E. Lorentz and A. Benallal. Gradient constitutive relations: numerical aspects and application to gradient damage. Comput. Meth. Appl. Mech. Eng., 194:5191-5220, 2005.

24. S. Mary, J. Vignollet, and R. de Borst. A numerical assessment of phase-field models for brittle and cohesive fracture: $\Gamma$-convergence and stress oscillations. Eur. J. Mech. A / Solids, 52:72-84, 2015.

25. C. Miehe, M. Hofacker, and F. Welschinger. A phasefield model for rate-independent crack propagation: Robust algorithmic implementation based on operator splits. Comput. Method. Appl. M, 199:2765-2778, 2010.

26. C. Miehe, F. Welschinger, and M. Hofacker. Thermodynamically consistent phase-field models of fracture: Variational principles and multi-field fe implementations. Int. J. Numer. Meth. Eng, 83(10):1273-1311, 2010.

27. N. Moës, J. Dolbow, and T. Belytschko. A finite element method for crack growth without remeshing. Int. J. Numer. Meth. Eng, 46:131-150, 1999.

28. T.T. Nguyen, J. Yvonnet, Q.-Z. Zhu, M. Bornert, and C. Chateau. A phase field method to simulate crack nucleation and propagation in strongly heterogeneous materials from direct imaging of their microstructure. Eng. Fract. Mech, 139:18-39, 2015.

29. T.T. Nguyen, J. Yvonnet, Q.-Z. Zhu, M. Bornert, and C. Chateau. A phase-field method for computational modeling of interfacial damage interacting with crack propagation in realistic microstructures obtained by microtomography. Comput. Meth. Appl. Mech. eng., 2016.

30. R.H.J. Peerlings, R. de Borst, W.A.M. Brekelmans, and H.P.J. de Vree. Gradientenhanced damage for quasi-brittle materials. Int. J. Num. Meth. Eng., 39(39):33913403, 1996.

31. K. Pham, J.-J. Marigo, and C. Maurini. The issues of the uniqueness and the stability of the homogeneous response in uniaxial tests with gradient damage models. J. Mech. Phys. Solids, (6), 2011.

32. S. Pietruszczak and S. Mroz. Finite element analysis of deformation of strain-softening materials. Int. J. Num. Methods Eng., 17:327-334, 1981.

33. G. Pijaudier-Cabot and Z. Bažant. Nonlocal damage theory. J. Eng. Mech., 113:15121533, 1987.

34. R. Romani. Rupture en compression des structures hétérogènes á base de materiaux quasi-fragiles. PhD thesis, Université Pierre et Marie Curie, 2013.

35. R. Romani, M. Bornert, D. Leguillon, R.L. Roy, and K. Sab. Detection of crack onset in double cleavage drilled specimens of plaster under compression by digital image correlation-theoretical predictions based on a coupled criterion. Eur. J. Mech. A-Solid, $51: 172-182,2015$.

36. C.G. Sammis and W.F. Ashby. The failure of brittle porous solids under compressive stress states. Acta Metall., 34(3):511-26, 1986.

37. R. Spatschek, M. Hartmann, E. Brener, K.H. Müller, and K. Kassner. Phase field modeling of fast crack propagation. Phys. Rev. Lett, 96(1):015502, 2006.

38. N. Triantafyllidis and E.C. Aifantis. A gradient approach to localization of deformation: I. hyperelastic materials. J. Elast., 16:225-237, 1986.

39. R.H.C. Wong, P. Lin, and C.A. Tang. Experimental and numerical study on splitting failure of brittle solids containing single pore under uniaxial compression. Mech. Mater. $38: 142-159,2006$

40. X.-P. Xu and A. Needleman. Numerical simulation of fast crack growth in brittle solids. J. Mech. Phys. Solids, 42(9):1397-1434, 1994.

41. F. Zhou and J.F. Molinari. Dynamic crack propagation with cohesive elements: a methodology to address mesh dependency. Int. J. Numer. Meth. Eng., 59:1-24, 2004. 